\title{
LATTICES OF CONVEX SETS
}

BY

\author{
MARY KATHERINE BENNETT
}

\begin{abstract}
If $V$ is a vector space over an ordered division ring, $C$ a convex subset of $V$ and $L$ the lattice of convex subsets of $C$, then we call $L$ a convexity lattice. We give necessary and sufficient conditions for an abstract lattice to be a convexity lattice in the finite dimensional case.
\end{abstract}

1. Introduction. In this paper we give necessary and sufficient conditions on a lattice $L$ so that $L$ is the lattice of convex subsets of some convex set in a finite dimensional vector space over an ordered division ring, and an extrinsic characterization of the face lattice of a convex polytope. Although some of the lattice theoretic terms to be used here are standard, others are not; we include now a collection of the necessary definitions and notational symbols to be used throughout the paper.

1.1 Definimion. Let $L$ be any lattice. $L$ is said to be atomistic iff $L$ is complete and for $x \in L, x \neq 0, x=\bigvee\{p \mid p \leqslant x$ and $p$ an atom of $L\}$.

1.2 Definirion. An atomistic lattice $L$ is said to be compactly atomistic iff whenever $p$ and $\left\{p_{i}\right\}_{i \in I}$ are atoms of $L$ with $p \leqslant \bigvee_{i \in I} p_{i}$, there is a finite subset $I_{0} \subseteq I$ with $p \leqslant \bigvee_{i \in I_{0}} p_{i}$.

1.3 RemarKs. It is well known that an atomistic lattice is compactly atomistic iff it is upper continuous.

1.4 Definition. Let $L$ be a lattice. The modular center of $L, \Re(L)$ is $\{x \in L: \Re(a, x)$ for all $a \in L\}$, cf. Definitions 2.3 and 2.4 of [1].

1.5 Notation. Any lattice we will consider has the property that $\mathfrak{R}(L)$ forms a meet-complete meet sublattice. For $a, b \in \Re(L)$ we will denote the join in $\Re(L)$ of $a$ and $b$ as $a \bar{V} b$.

1.6 Definition. Let $L$ be any lattice. The distributive center of $L, \mathscr{D}(L)=$ $\{x \in L \mid(a \vee b) \wedge x=(a \wedge x) \vee(b \wedge x)$ for all $a, b \in L\}$

1.7 Notation. In any lattice we will consider, $\mathscr{D}(L)$ will be closed under arbitrary meets. For $z \in L$ we will denote by $\mathscr{D}(z)$ the element $\wedge\{x \in$ D $(L): x \geqslant z\}$.

1.8 Definition. A lattice $L$ is said to be additive iff whenever $p$ is an atom

Presented to the Society, November 1, 1976; received by the editors February 9, 1976 and, in revised form, May 20, 1976.

AMS (MOS) subject classifications (1970). Primary 06A23, 52A05; Secondary 06A30, 06A35, 52A25, 52D05, 50D25. 
of $L$ with $p \leqslant a \bigvee b$, there exist atoms $a_{1} \leqslant a$ and $b_{1} \leqslant b$ such that $p<a_{1}$ $\vee b_{1}$.

1.9 Definition. A lattice $L$ is said to be $\mathscr{D}$-additive iff $\mathscr{D}(a \wedge b)=1$, implies that for any atom $p \leqslant a \vee b$, there are atoms $a_{1} \leqslant a, b_{1} \leqslant b$ such that $p \leqslant a_{1} \vee b_{1}$.

1.10 DefINITION. A projective geometry is a compactly atomistic modular lattice.

1.11 REMARK. It is well known that a projective geometry of height at least 4 is the lattice of subspaces of a vector space over a division ring.

1.12 Definition. It $L$ is a lattice with a well defined height function, an element of height 2 will be called a line in $L$.

1.13 Definition. If $C$ is a convex subset of vector space $V$ over ordered division ring $R$, a subset $D \subseteq C$ is called a face of $C$ iff whenever $a X+(1-$ a) $Y \in D$ with $0<a<1$ and $X, Y \in V$ then $X, Y \in D$. A singleton face is called an extreme point.

1.14 Definition. A convex set $C$ is called a polytope iff it has a finite number of extreme points which span $C$.

\section{Main Theorem.}

2.1 THEOREM. Let $L$ be a compactly atomistic additive lattice satisfying these conditions:

(i) For $a, b, c, d$, $e$ atoms of $L$ with $a \neq b$ and $a \vee b \leqslant d \vee e$, then $(a \vee b)$ $\wedge c=(a \vee c) \wedge b=(b \vee c) \wedge a=0$ iff $(d \vee e) \wedge c=(d \vee c) \wedge e=(e$ $\vee c) \wedge d=0$. Furthermore $a \leqslant d \vee b$ iff $a \wedge(e \vee b)=0$.

(ii) $\mathfrak{N}(L)$ is $\mathscr{Q}$-additive.

(iii) Given $x, y$ atoms of $L, \exists z \leqslant x \vee y, z \neq x, z \neq y, z$ an atom.

(iv) $4<h(\mathscr{T}(L))<\infty$.

(v) $l$ a line in $\mathscr{R}(L)$ such that $l \leqslant x \bar{\vee} y \bar{\vee} z ; x, y, z$ atoms; $l \wedge(x \vee y) \neq$ $0, x, y$ implies $[l \wedge(x \vee z)] \vee[l \wedge(y \vee z)] \neq 0$

Then there exists an ordered division ring $R$, a finite dimensional vector space $V$ over $R$, and a convex subset $C \subseteq V$ such that $L$ is the lattice of convex subsets of $C$.

Conversely, given $R, V$ and $C$ as above, with the dimension of $C$ not less than 4 , the lattice of convex subsets of $C$ is compactly atomistic, additive and satisfies (i) - (v).

The remainder of this section will be denoted to proving the theorem stated above. We shall assume throughout that $L$ will denote a compactly atomistic additive lattice. In the following lemma, we collect some results which have been proved in $\S 3$ of [1], or which will be proved in $\S 3$ of this paper.

2.2 LEMMA.

(i) $\Re(L)$ is closed under arbitrary meets. 
(ii) $\mathscr{N}(L)=\left\{x \mid x \in L\right.$ and whenever $x_{1}, x_{2} \leqslant x$ are distinct atoms with $x_{1}<x_{2} \vee y$ and $y$ an atom, then $\left.y \leqslant x\right\}$.

(iii) Tर $(L)$ is compactly atomistic.

(iv) If $L$ satisfies 2.1(i) then for $a, b, c$ atoms of $L, c \leqslant a \bar{\vee} b$ iff $c<a \vee b$, $a \leqslant b \vee c$ or $b \leqslant a \vee c$.

(v) If $L$ satisfies 2.1(i) then for $p$ an atom of $L, \mathfrak{N}(L)(p, 1)$ is compactly atomistic.

(vi) $\mathscr{D}(L)$ is closed under arbitrary meets. (See 3.2. below.)

2.3 LEMMA. Let $L$ satisfy 2.1(i) and (ii). Then for $p$ an atom of $L$ such that $\mathscr{D}(p)=1$, $\Re(L)(p, 1)$ is a projective geometry.

Proof. By $2.2(v)$ we need only prove $\mathscr{R}(L)(p, 1)$ is modular and complemented.

Let $c, a, b \in \mathfrak{R}(L)(p, 1)$ with $c \leqslant b$. As always $c \bar{\vee}(a \wedge b) \leqslant(c \bar{\vee} a) \wedge$ $b$. Let $t \leqslant(c \bar{\vee} a) \wedge b, t$ an atom. Now $\mathscr{D}(\mathscr{N}(L)) \subseteq \mathscr{D}(L)$, hence $\mathscr{D}(p)=1$ in $\mathscr{R}(L)$. By the $\mathscr{D}$-additivity of $\mathscr{R}(L)$ since $p \leqslant c \wedge a, t \leqslant c_{1} \bar{\vee} a_{1}$ where $c_{1}$ and $a_{1}$ are atoms under $c$ and $a$ respectively. If $t=c_{1}$ then $t<c<\bar{V}(a \wedge$ $b)$ and we are done. If $t \neq c_{1}$, then $c_{1} \bar{V} a_{1}=c_{1} \bar{V} t$ by 2.2(iv). Thus $a_{1} \leqslant c_{1} \bar{\vee} t \leqslant b$, and $t \leqslant c_{1} \bar{\vee} a_{1} \leqslant c \bar{\vee}(a \wedge b)$ so modularity obtains.

2.4 LEMMA. If $L$ satisfies 2.1 (i)-(iv) there is an atom $p \in L$ such that O) $(p)=1$.

Proof. If $x, y$ and $z$ are distinct atoms of $L$ with $y<x \vee z$ then $\mathscr{D}(y)=$ $\mathscr{D}(x \vee z)$. Clearly $\mathscr{D}(y) \leqslant \mathscr{D}(x \vee z)$ so only the reverse inclusion needs to be proved. Now $(x \vee z) \wedge \mathscr{D}(y)=(x \wedge \mathscr{D}(y)) \vee(z \wedge \mathscr{D}(y))$. But on the one hand $y \leqslant(x \vee z) \wedge \mathscr{D}(y)$; on the other hand $(x \wedge \mathscr{D}(y)) \vee(z \wedge$ $\mathscr{D}(y))=0, x, z$ or $x \vee z$. Since $y \nless 0, x$ or $z$,

$$
(x \vee z) \wedge \mathscr{D}(y)=(x \wedge \mathscr{D}(y)) \vee(z \wedge \mathscr{D}(y))=x \vee z
$$

and $x \leqslant \mathscr{D}(y), z \leqslant \mathscr{D}(y)$. Thus $x \vee z \leqslant \mathscr{D}(y)$ and $\mathscr{D}(x \vee z) \leqslant \mathscr{D}(y)$ so equality obtains. Now let $q_{1}$ be an atom of $L$. If $\mathscr{D}\left(q_{1}\right)=1$ we are done. Otherwise let $s_{1}$ be an atom such that $s_{1} \nless \mathscr{D}\left(q_{1}\right)$. Let $q_{2} \leqslant q_{1} \vee s_{1}, q_{2} \neq$ $q_{1}, s_{1}$. $\mathscr{D}\left(q_{1}\right)<\mathscr{D}\left(q_{1} \vee s_{1}\right)=\mathscr{D}\left(q_{2}\right)$. Similarly we can find $\mathscr{D}\left(q_{3}\right)>\mathscr{D}\left(q_{2}\right)$ and obtain a chain $\mathscr{D}\left(q_{1}\right)<\mathscr{D}\left(q_{2}\right)<\cdots<\mathscr{D}\left(q_{n}\right)$. But $\mathscr{D}\left(q_{i}\right) \in \mathscr{D}(L) \subseteq$ $\mathfrak{R}(L)$ and $h(\mathfrak{R}(L))<\infty$ so the chain is finite. It is easy to see that its largest element is 1 , so we are done.

The two preceding lemmas immediately yield this corollary.

2.5 Corollary. Let L satisfy 2.1(i)-(iv). Then there is an atom $p \in L$ such that $\mathfrak{T}(L)(p, 1)$ is a projective geometry of height greater than or equal to 3.

It is well known that such a geometry is the lattice of subspaces of a vector 
space over a division ring. We next show that the division ring in question is ordered.

2.6 LemMA. Let $L$ satisfy the hypotheses of 2.1. If $p$ is an atom of $L$ such that $\mathcal{D}(p)=1$, then whenever $a$ is an atom of $L, a \neq p$, there exists an atom $b \in L, b \neq p$ such that $p \leqslant a \vee b$.

Proof. We set $S=\{x: x$ an atom of $L$ and $x=p$ or $x \neq p$ and there is $y \neq p$ with $p \leqslant x \vee y\}$, and show by heavy use of 2.1(v) that the atoms under $\bar{V} S$ are exactly those in $S$. We next show that $\bar{V} S$ is in $D(L)$ and the result follows.

2.7 LEMMA. Let $L$ satisfy the hypotheses of 2.1. Let $p$ be an atom of $L$ such that $\mathscr{D}(p)=1$. Then $\mathscr{N}(L)(p, 1)$ is the lattice of subspaces of a vector space $V$ over an ordered division ring $R$.

Proof. Select and fix an atom $z \neq p$. Next select and fix an atom $q$ such that $q \leqslant z \bar{\vee} p$, and an atom $e$ such that $e \leqslant z \vee q, e \neq z, e \neq q$. Let $A=\{t \mid t$ an atom of $L,(z \vee t) \wedge(p \bar{\vee} q)=0$ and $t \leqslant z \bar{\vee} p \bar{\vee} q\}$. For $t \in A$, define $A_{t}=\{x \mid x$ an atom of $L$ and $t<p \vee x$ or $x \leqslant p \vee t\}$. It can be shown by considering several cases that $A_{t}$ is the set of atoms under an element of $L$ i.e., if $a$ and $b$ are atoms in $A_{t}$ and $c$ is an atom such that $c \leqslant a \vee b$, then $c$ is in $A_{t}$ as well. Thus from now on we will refer to $\bigvee\left\{x \mid x \in A_{t}\right\}$ as $p \bigvee t$, and if $x$ is an atom under $p \bigvee t$, then either $x<p \bigvee$ $t$ or $t<p \vee x$. We next note that for $t, s \in A,(t \vee s) \wedge(p \bar{\vee} q)=0$. This is so because by definition of $A$ neither $t$ nor $s$ is under $p \bar{V} q$. But if $(t \vee s) \wedge(p \bar{\vee} q)=v$ where $v$ is an atom unequal to $t$ or $s$, by 2.1(v) either $(t \vee z) \wedge(p \bar{\vee} q) \neq 0$ or $(s \vee z) \wedge(p \bar{\vee} q) \neq 0$. Both of these possibilities lead to contradictions since $s$ and $t$ are in $A$. We can further show that $A$ is the set of atoms under some lattice element, for if $t_{1}, t_{2} \in A$ with $t_{3} \leqslant t_{1} \vee t_{2}$, then $t_{3} \in A$. Again this is proved by several cases making heavy use of $2.1(\mathrm{v})$.

We can next show that for $t \in A$ exactly one of three conditions holds:

$$
\begin{array}{ll}
(t \vee q) \wedge(p \check{\vee} e)=0, & (t \vee q) \wedge(p \check{\vee} z)=0 . \\
(t \vee q) \wedge(p \check{\vee} e) \neq 0, & (t \vee q) \wedge(p \vee z)=0 . \\
(t \vee q) \wedge(p \vee e) \neq 0, & (t \vee q) \wedge(p \vee z) \neq 0 .
\end{array}
$$

This is so because if $(t \vee q) \wedge(p \vee \bar{\vee})=0$ but there is an atom $k=(t \vee$ q) $\wedge(p \vee z)$, then either $k \leqslant p \vee z$ or $z \leqslant p \vee k$. If $k=z$ then $z<t \vee q$. But $e \leqslant z \vee q$ so $e \leqslant t \vee q$. Thus $z=k=e$, a contradiction. If $k=p$ then $p, q \leqslant t \vee q$ so $t \leqslant p \bar{\vee}$, again a contradiction. Thus if $k \leqslant p \vee z$ exists it is unequal to $p$ or $z$ so we can make use of $2.1(v)$, namely $(p \vee e) \wedge(k \vee z)$ $\neq 0$ or $(p \vee e) \wedge(k \vee q) \neq 0$. In the first instance $(p \vee e) \wedge(k \vee z)$ must equal $p$, so $p \leqslant k \vee z$ which is impossible since $k \leqslant p \bigvee z$. In the second 
instance if $(p \bar{\vee} e) \wedge(k \vee q)=r$, then since $r \leqslant k \vee q$ and thus $r \in A$. Thus $r<p \vee e$ and $r \leqslant(p \vee e) \wedge(t \vee q)$, a contradiction. A similar sort of argument shows that if $z<p \vee k$, then $(p \vee e) \wedge(t \vee q) \neq 0$ and therefore exactly one of (1), (2) and (3) holds. If condition (1) holds we will say $e \rightarrow t$; in case of (2) we will say $z \rightarrow t \rightarrow e$ and (3) will be written $t \rightarrow z$. Again making use of 2.1(v) we can show that if $s$ is an atom with $s \leqslant t \vee p$, (1), (2) or (3) holds for $s$ iff the same condition holds for $t$. Thus we can define

$p \check{V} e \rightarrow p \check{V} t$ if (1) holds for $t$,

$p \check{V} z \rightarrow p \check{V} t \rightarrow p \check{V} e$ if (2) holds for $t$, or

$p \check{V} z \rightarrow p \breve{V} t$ if (3) holds for $t$,

and have an ordering on the elements of the form $p \check{V} t$ for $t \in A$. We shall define $\mathcal{P}$ to be $\{p \vee t \mid(1)$ or (2) holds for $t\}=\{p \bigvee t \mid(q \vee t) \wedge(p \bigvee z)=$ $0\}$. By 2.6 we find that the elements of the form $p \breve{V} t$ for $t$ an atom in $A$ correspond to the elements of the coordinatizing division ring where we shall take $p \bar{V} q$ as the "point at infinity" with $p \check{V} e$ as the identity [2]. It is well known that the division ring obtained is independent of the choices of the zero, identity and subspace at infinity [2], so making these choices does not affect what follows. We will show that $\mathscr{P}$ satisfies the conditions for the positive elements of an ordered division ring. In order to do this we need to recall the processes of addition and multiplication in this setting.

We first select $s \leqslant p \bar{V} q \bar{V} z$, with $s$ an atom. We shall refer to $p \bar{V} q \bar{V} s$ as $\pi$-it will play the role of a subspace at infinity. Let $a$ and $c$ be elements of $A$ such that $p \check{V} a$ and $p \check{V} c$ are in $\mathcal{P}$. Without loss of generality we may assume $(p \check{V} a) \wedge(q \vee z)=a$ and $(p \check{V} c) \wedge(q \vee z)=c$, and that $a \leqslant z$ $\vee c$. To find $(p \check{V} z)+(p \check{V} c)$ we first select $u$, an atom under $\pi$ such that $u \leqslant p \bar{\vee} q$. Let $t \leqslant u \vee c ; t \neq u, e ; t$ an atom. By 2.1(v) there is an atom $v$ such that $(z \bar{V} t) \wedge(u \vee q)=v$. By considering the various possibilities and using 2.1(v) we show $t \leqslant z \vee v$. Next by similar methods we prove that there is an atom $y$ such that $y=(t \bar{\bigvee} q) \wedge(a \vee v)$, and that $y<t \vee q$. Again we show that there is an atom $r$ with $(u \bigvee y) \wedge(a \vee q)=r$. It follows from the coordinatization method in [2] that $p \check{V} r$ is $(p \check{V} a)+(p \check{V} c)$. Also it is clear that $p \vee r \in \mathcal{P}$ since $r \leqslant a \vee q \leqslant z \vee q$ so $(p \vee z) \wedge(q \vee r)=0$. Thus the sum of elements in $\mathscr{P}$ is again in $\mathcal{P}$. We shall just sketch the proof that $\mathcal{P}$ is closed under products-and make the remarks that all letters introduced are atoms which can be shown to exist. Let $a, c$ and $u$ be as above with $a<e \vee c$. Let $t \leqslant u \vee c ; t \neq u, c$. Then $(e \bar{\vee} t) \wedge(u \vee q)=v$ and $t \leqslant e \vee v$. Now $(z \bar{\vee} t) \wedge(a \vee v)=y$ and $t=(z \vee y) \wedge(e \vee v)$. Now $(u$ $\bar{\vee} y) \wedge(a \vee q)=r$ and $p \check{V} r=(p \vee a)(p \check{V} c)$. Since $r \leqslant a \vee q, p \check{V} r \in$ $\mathcal{P}$. In similar fashion we show that $(p \vee a)(p \vee c) \in \mathcal{P}$ when $e<a \vee c$, and $a, c<z \vee e$. Thus $\mathcal{P}$ is closed under multiplication. We further show that if $z<a \vee q$, then $-(p \vee a)=p \check{V} x$ where $x \leqslant z \vee q$ which yields the 


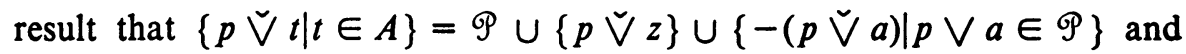
our division ring is ordered.

At this stage we have shown that for any lattice $L$ satisfying the hypotheses of 2.1 , there is an atom $p \in L$ such that $\mathscr{N}(L)(p, 1)$ is the lattice of subspaces of a finite dimensional vector space over an ordered division ring. We next look at the order relation $\rightarrow$ in a slightly different way with a view to obtaining a connection between the order relation on $L$ and the ordering of the ring. In the remainder of this section we shall assume $L$ satisfies the hypotheses of Theorem 2.1.

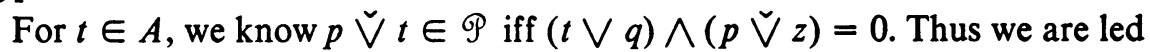
to make this definition.

\subsection{Definition. For $t, t^{\prime} \in A$ we say $t \rightarrow t^{\prime}$ iff $\left(t^{\prime} \vee q\right) \wedge(p \check{V} t)=0$}

By methods similar to those employed in Lemma 2.7 it is not difficult to show that a trichotomy law obtains, namely that for $t, t^{\prime} \in A$ exactly one of $p \breve{V} t=p \grave{V} t^{\prime}, t \rightarrow t^{\prime}$, or $t^{\prime} \rightarrow t$ holds. Furthermore the current definition of $\rightarrow$ does not depend on particular elements in $p \breve{V} t$ as the following lemma shows.

\subsection{Lemma. Let $y<p \check{V} t$ and $t^{\prime} \rightarrow t$. Then $t^{\prime} \rightarrow y$.}

Proof. $(t \vee q) \wedge\left(p \vee t^{\prime}\right)=0$. Now if $y \rightarrow t^{\prime}$ we would have $\left(t^{\prime} \vee q\right) \wedge(p$ $\check{\vee} y)=0$. But $\left(t^{\prime} \vee q\right) \wedge(p \vee y)=\left(t^{\prime} \vee q\right) \wedge(p \vee t) \neq 0$, a contradiction. If $p \breve{V} y=p \breve{V} t^{\prime}$, then $p \vee t=p \breve{V} t^{\prime}$, again a contradiction. Thus the only possibility is $t^{\prime} \rightarrow y$.

2.10 Lemma. If $t, u, w \in A$ with $t \rightarrow u$ and $u \rightarrow w$, then $t \rightarrow w$.

Proof. We shall sketch the proof of this result, which depends heavily on 2.1(v). We need to show that $(w \vee q) \wedge(p \vee t)=0$. We obtain contradictions in case the meet above equals $w$ or $q$, so we can assume that if it not zero, it is $x \leqslant w \vee q, x \neq w, q$. By $2.1(\mathrm{v})$ we can show that $(p \bar{\vee} t) \wedge(w \vee$ $u)=y$. Repeated use of (v) yields contradictions if $y<p \vee x$ or if $x<p \vee$ $y$. But if $x$ exists, it is in $A$, as is $y$, so we get $(w \vee q) \wedge(p \vee t)=0$.

What we now have is an ordering which can easily be made into a total ordering on our division ring such that $\mathscr{P}$, as defined before, is the set of positive elements.

2.11 Lemma. Let $a, b, c \in A$ with $a \leqslant b \vee c$. Then if $b \rightarrow c, b \rightarrow a \rightarrow c$.

Proof. By trichotomy we know that $(c \vee q) \wedge(p \vee b)=0$ and $(p \vee c)$ $\wedge(b \vee q)=t$ for some atom $t$. Thus $(p \vee t) \wedge(a \vee q) \neq 0$ or $(p \vee t) \wedge(a$ $\vee b) \neq 0$. The second possibility contradicts $a \leqslant b \vee c$ so $(p \vee t) \wedge(q \vee a)$ $\neq 0$. Thus $(p \vee c) \wedge(q \vee a) \neq 0$ and $a \rightarrow c$. A similar sort of argument show $b \rightarrow a$. We should note that we have used in this proof, one result 
immediately obtained from trichotomy, namely that for $x, y$ atoms of $A, x \leqslant$ $p \check{V} y$,

$$
(p \breve{V} x) \wedge(q \vee y)=0 \text { iff }(p \check{V} y) \wedge(q \vee x) \neq 0
$$

Having obtained $\Re(L)(p, 1)$ as the lattice of subspaces of a finite dimensional vector space over an ordered division ring $R$, we now cease to refer to the elements of $R$ as elements of the form $p \vee t$ for $t \in A$, but will use Greek letters instead.

We now state without proof a result about vector spaces coordinatized by ordered division rings.

2.12 LEMMA. Let $V$ be a vector space over an ordered division ring $R$. Let $\{\overline{0, X, Y}\}$ be a plane in that space. Then $R$ is isomorphic to $\{l(0, Z) \mid l(0, Z) \neq$ $l(0, X)$ and $Z \in\{\overline{0, X, Y}\}\}$. This isomorphism is given by $f(\alpha)=l(0, \alpha X+$ $Y)$. Clearly if $\alpha$ is between 0 and 1 in $R$, then $f(\alpha)$ is of the form $l(0, \beta X+(1$ $-\beta) Y$ ) with $\beta$ strictly between 0 and 1 and conversely.

2.13 Proof of 2.1. Select and fix an atom $p \in L$ such that $\mathscr{D}(p)=1$. Since by previous results $\Re(L)(p, 1)$ is the lattice of subspaces of a finite dimensional vector space $V$ over an ordered division ring $R$, we can select $\left\{X_{1}, \ldots, X_{n}\right\}$ as a basis for $V$. Then $l\left(0, X_{i}\right)$ corresponds to $p \bar{V} x_{i}$ where $x_{1}, \ldots, x_{n}$ are atoms of $L$. We shall rename the atoms of $L$ as vectors in $V$. Let $p=0 \in V$ and $x_{1}=X_{1}$. Let $x_{2}=\alpha X_{2}$ where $\alpha$ is an undetermined element of $R$. If $p \bar{V} s$ corresponds to $l\left(0, X_{1}+X_{2}\right)$ we can choose our $x_{2}$ in such fashion that $(p \bar{\vee} s) \wedge\left(x_{1} \vee x_{2}\right) \neq 0$. For $t \leqslant x_{1} \bar{\vee} x_{2}, t \neq x_{1}, x_{2}$ then $p \bar{V} t$ corresponds to $l\left(0, X_{1}+\beta X_{2}\right)$ for some known $\beta \in R$. We set

$$
t=\left(1+\beta \alpha^{-1}\right)^{-1}\left(X_{1}+\beta X_{2}\right)=\left(1+\beta \alpha^{-1}\right)^{-1}\left(X_{1}+\beta \alpha^{-1}\left(\alpha X_{2}\right)\right)
$$

and refer to $t$ as $t_{1}(\alpha) X_{1}+t_{2}(\alpha) X_{2}$. By the ordering of $R$ done previously using 2.11 and 2.12 we find that if $t \leqslant s \vee r \leqslant x_{1} \bar{\vee} x_{2}$ then

$$
\begin{aligned}
& t_{1}(\alpha) X_{1}+t_{2}(\alpha) \alpha^{-1}\left(\alpha X_{2}\right) \\
& \quad=\mu\left(s_{1}(\alpha) X_{1}+s_{2}(\alpha) \alpha^{-1}\left(\alpha X_{2}\right)\right)+(1-\mu)\left(r_{1}(\alpha) X_{1}+r_{2}(\alpha) \alpha^{-1}\left(\alpha X_{2}\right)\right),
\end{aligned}
$$

where $0 \leqslant \mu \leqslant 1$ in $R$, i.e., that $t$ is a convex combination of $s$ and $r$. We observe that if $\left\{X_{1}, \ldots, X_{n}\right\}$ is a basis for $V$, so is $\left\{\left(1+\alpha^{-1}\right)^{-1}\left(X_{1}+\right.\right.$ $\left.\left.X_{2}\right), X_{2}, \ldots, X_{n}\right\}$. We let $y=\left(x_{1} \vee x_{2}\right) \wedge(p \bar{\vee} w)$ where $w$ is an atom with $p \vee w$ corresponding to $l\left(0,\left(1+\alpha^{-1}\right)^{-1}\left(X_{1}+X_{2}\right)\right)$. We take $t \leqslant\left(y \vee x_{2}\right), t$ $\neq y, x_{2}$, and note that $t$ has been set equal to $\left(1+\beta \alpha^{-1}\right)^{-1}\left(X_{1}+X_{2}\right)$ for some known $\beta$. However repeating the process above with the new basis, $t$ is set equal to $\left(1+\gamma \alpha^{-1}\right)^{-1}\left(\left(1+\alpha^{-1}\right)^{-1}\left(X_{1}+X_{2}\right)+\gamma X_{2}\right)$ for some known $\gamma$. The two expressions for $t$ enable us to find $\alpha$ and the determation of $\alpha$ can be shown to be independent of $t$ by 2.11 and 2.12. If $s \leqslant p \vee x_{2}$ we will set $s=\delta X_{2}$ and find $\delta$ as we found $\alpha$. 
For $s<p \vee x_{1}, s \neq p, x_{1}$, we select $\delta X_{2} \leqslant p \vee \alpha X_{2}$ and consider $(s \vee$ $\left.\alpha X_{2}\right) \wedge\left(X_{1} \vee \delta X_{2}\right)=w$. Now $p \bar{\vee} w=l\left(0, X_{1}+\beta X_{2}\right)$ for some known $\beta$, so we can compute in terms of $\delta, w$ as $w_{1}(\delta) X_{1}+w_{2}(\delta) X_{2}$. Then we compute $l\left(\alpha X_{2}, w_{1}(\delta) X_{1}+w_{2}(\delta) X_{2}\right) \cap l\left(0, X_{1}\right)$ and obtain $s$ as $s(\delta) X_{1}$. Again the ring order yields $0 \leqslant s(\delta) \leqslant 1$, and $s(\delta)$ is independent of the choice of $t$. Furthermore we note that for $w \leqslant p \vee x_{1} \bigvee x_{2}, w$ can be represented as $\rho X_{1}+\mu \alpha X_{2}$, with $0 \leqslant \rho, \mu \leqslant 1$. Also if $0 \leqslant s \leqslant t \vee r \leqslant p \vee x_{1} \vee x_{2}$, then the representation of $s$ is a convex combination of the representations of $t$ and $r$.

If $q$ is an atom of $L$ such that $q \leqslant p \bar{V} x_{1} \bar{V} x_{2}$ with $q \leqslant x_{1} \bar{V} x_{2}$, then for $x \leqslant x_{1} \bigvee x_{2}, s \neq x_{1}, x_{2},(q \bar{\vee} s) \wedge\left(p \vee x_{1}\right) \neq 0$ or $(q \bar{\vee} s) \wedge\left(p \vee x_{2}\right) \neq 0$. Also $p \bar{V} q=l\left(0, X_{1}+\beta X_{2}\right)$ for some known $\beta$ or $p \bigvee q=l\left(0, X_{2}\right)$ if $q \leqslant p$ $\bar{\vee} x_{2}$. Using this information we get $q$ as a linear combination of $0, X_{1}, X_{2}$. Again $q<r \vee s$ implies that $q$ will be represented as a convex combination of the representations of $r$ and $s$. Thus the atoms under $p \bar{V} x_{1} \bar{V} x_{2}$ are representable as the vectors in a convex subset of the plane in $V$ determined by $X_{1}$ and $X_{2}$.

We now repeat the above process using $X_{1}$ and $\alpha^{\prime} X_{3}$ for some $\alpha^{\prime} \in R$. By selecting $q \leqslant p \vee x_{1}$, we already have $q$ represented as $\gamma X_{1}$. Thus we can easily compute $\alpha^{\prime}$.

We continue this process, choosing $x_{i}$ so that $p \bar{V} x_{i}$ is $l\left(0, X_{i}\right)$ and obtain each atom $q$ in $L$ represented as a linear combination of the $X_{i}$ with $q \leqslant r \vee s$ yielding $q$ as a convex combination of $r$ and $s$.

Conversely, given $r$ and $s$ as the expressions, any convex combination of these will be the expression for an atom under $r \vee s$, and the atoms of $L$ can be represented as the vectors in a convex subset $C$ of $V$ with the line segments being the joins of pairs of atoms.

By compact atomisticity and additivity we can show that the vectors in any convex subset of $C$ are exactly the atoms under some element of $L$, and, conversely, obtaining $L$ as the lattice of convex subsets of $C$.

Routine linear algebra arguments show that if $V$ is a vector space over ordered division ring $R$ with $C$ a convex subset of $V$, then the lattice of convex subsets of $C$ satisfies the lattice theoretic hypotheses of Theorem 2.1 so the proof is complete.

3. Distributive centers of additive lattices. We collect here some elementary results on distributive centers with a view to obtaining an extrinsic characterization of the face lattice of a convex polytope.

3.1 Lemma. Let $L$ be any lattice. Then if $z$ and $w$ are elements in $\mathscr{D}(L)$, $z \wedge w \in \mathscr{D}(L)$.

Proof. Let $x, y \in L$. Then 


$$
\begin{aligned}
&(x \wedge z \wedge w) \vee((y \wedge z \wedge w)=((x \wedge z) \vee(y \wedge z)) \wedge w \\
&=((x \vee y) \wedge z) \wedge w=(x \vee y) \wedge(z \wedge w)
\end{aligned}
$$

3.2 THEOREM. If $L$ is compactly atomistic and additive, then $\mathscr{D}(L)$ is closed under arbitrary meets.

Proof. Let $\left\{z_{0}\right\}_{i \in I} \subseteq \mathscr{D}(L)$ with $x$ and $y$ elements of $L$. Let $t$ be an atom under $(x \vee y) \wedge\left(\bigwedge z_{i}\right)$. Then $t \leqslant x_{1} \vee y_{1}$ where $x_{1}$ and $y_{1}$ are atoms under $x$ and $y$ respectively. Thus $t \leqslant\left(x_{1} \vee y_{1}\right) \wedge z_{i}$ for any $i$ and then $t<\left(x_{1} \wedge z_{i}\right)$ $\vee\left(y_{1} \wedge z_{i}\right)$. If $x_{1} \wedge\left(\wedge z_{i}\right)=0$ then $x_{1} \wedge z_{0}=0$ for some $z_{0}$, so $t<y_{1} \wedge z_{0}$ and thus $t=y_{1}$ and $t \leqslant y_{1} \wedge\left(\wedge z_{i}\right) \leqslant\left(x \wedge \wedge z_{i}\right) \vee\left(y \wedge \wedge z_{i}\right)$. If $x_{1} \wedge$ $\left(\wedge z_{i}\right)=x_{1}$ then $x_{1} \leqslant \wedge z_{i}$ and $x_{1} \vee\left(y_{1} \wedge \wedge z_{i}\right)=\left(x_{1} \vee y_{1}\right) \wedge\left(\wedge z_{i}\right)$ by 2.2(i) since $\mathscr{D}(L) \subseteq \pi(L)$. Thus $t \leqslant\left(x_{1} \vee y_{1}\right) \wedge\left(\wedge z_{i}\right)$ implies $t<\left(x_{1} \wedge\right.$ $\left.\left(\wedge z_{i}\right)\right) \vee\left(y_{1} \wedge\left(\wedge z_{i}\right)\right) \leqslant\left(x \wedge\left(\wedge z_{i}\right)\right) \vee\left(y \wedge\left(\wedge z_{i}\right)\right)$ and $(x \vee y) \wedge\left(\wedge z_{i}\right)$ $<\left(x \wedge\left(\wedge z_{i}\right) \vee\left(y \wedge\left(\wedge z_{i}\right)\right)\right.$. Since the reverse inclusion always holds, $\mathbb{D}(L)$ is closed under arbitrary meets.

3.3 COROLlaRY. Under the hypotheses of 3.2, $D(L)$ is a complete lattice in its own right, and is a meet-sublattice of $L$.

3.4 TheOREM. Let $L$ be additive and atomistic. Then $z \in \mathscr{D}(L)$ iff $(p \vee q)$ $\wedge z=(p \wedge z) \vee(q \wedge z)$ for all atoms $p$ and $q$ in $L$.

Proof. One implication is trivial. Let $(p \vee q) \wedge z=(p \wedge z) \vee(q \wedge z)$ for all atoms $p, q$ in $L$. Let $x, y \in L$ and let atom $t \leqslant(x \vee y) \wedge z$. By additivity there are atoms $x_{1} \leqslant x, y_{1} \leqslant y$ so that

$$
t=\left(x_{1} \vee y_{1}\right) \wedge z=\left(x_{1} \wedge z\right) \vee\left(y_{1} \wedge z\right)<(x \wedge z) \vee(y \wedge z)
$$

As in the proof of 3.2, $z \in \mathscr{D}(L)$.

3.5 COROLlary. Let $L$ be the lattice of convex subsets of $C$ a convex subset of vector space $V$ over ordered division ring $R$. For $w \in L, w$ is a face of $C$ iff $w \in \mathscr{D}(L)$.

Proof. Let $w \in \mathscr{D}(L)$. Let $p, q$ be atoms of $L$ such that $(p \vee q) \wedge w \neq$ $0, p, q$. Then $(p \wedge w) \vee(q \wedge w) \neq 0, p, q$. If $t \neq p, q$ is an atom under $(p \wedge w) \vee(q \wedge w)$, then if $p \wedge w=0, t \leqslant q \wedge w$ and $t=q$. Similarly if $q \wedge w=0, t=p$. Thus $p \wedge w \neq 0 \neq q \wedge w$, and $p=p \wedge w$ with $q=q \wedge$ $w$. Thus $(p \vee q) \wedge w=(p \wedge w) \vee(q \wedge w)=p \vee q$, and by definition $w$ is a face of $C$.

Conversely if $w$ is a face of $C$, a simple argument using Theorem 3.4 shows that $w \in \mathscr{D}(L)$.

3.6 COROLlaRy. If $L$ is as in 3.5, then the extreme points of $C$ are exactly those atoms of $L$ which are $D(L)$. 
The proof is immediate.

3.7 THEOREM. $M$ is the face lattice of a convex polytope iff $M=\mathscr{D}(L)$ where $L$ satisfies the hypotheses of 2.1 and there exist atoms $p_{1}, \ldots, p_{n} \in \mathscr{D}(L)$ such that $p_{1} \vee \cdots \vee p_{n}=1$.

The proof follows from the results of $\$ 2$ and Corollary 3.5.

4. Conclusions. In $\$ 2$, we characterized lattices of convex subsets of convex sets. This characterization was limited to those convex sets whose linear spans are finite dimensional, since we needed to have an atom $p$ such that $\mathscr{D}(p)=$ 1-namely a nonempty algebraic interior. The characterization theorem still holds if we drop the finite dimensionality assumption, and replace it with the existence of an atom as described above. It would be interesting however to characterize lattices of convex subsets of convex sets in general-this may be possible by using categorical constructions on those sublattices which satisfy our dimensionality condition.

Furthermore it would be interesting to give intrinsic conditions on a lattice $L$ which would insure that it is the distributive center of a lattice satisfying the hypotheses of Theorem 2.1-some categorical tools might again be of use here.

\section{BIBLIOGRAPHY}

1. M. K. Bennett, On generating affine geometries, Algebra Universalis 4 (1974), 207-219. MR 50 \#4417.

2. Coordinatization of affine and projective space, Discrete Math. 4 (1973), 219-231. MR 47 \#948.

Department of Mathematics and Statistics, University of MassachusetTs, amherst, MASSACHUSETTS 01003 